Case Reports

Rajan K Sharma, MBBS, MS

Department of Neurosurgery

Bir Hospital, NAMS

Kathmandu, Nepal

Gopal R Sharma, MS

Department of Neurosurgery

Bir Hospital, NAMS

Kathmandu, Nepal

Prakash Bista , MCh

Department of Neurosurgery

Bir Hospital, NAMS

Kathmandu, Nepal.

Rajiv Jha, MS, MCh

Department of Neurosurgery

Bir Hospital, NAMS

Kathmandu, Nepal.

Address of correspondence:

Rajan K Sharma, MBBS, MS

Department of Neurosurgery

Bir Hospital, NAMS

Kathmandu, Nepal

Email: Rajanktm58@gmail.com

Received, 6 January, 2017

Accepted, 25 January, 2017
Nepal Journal of Neuroscience 14: 36-39, 2017

\section{Carotid Cavernous Fistula: \\ Role of Open Surgery in \\ The Era of Endovascular \\ Treatment}

A 18-year-old right handed girl presented with progressive proptosis, chemosis, diplopia and an orbital bruit of right eye, 3 days after alleged history of fall from a tree. The intraocular pressure was increased. The suspected diagnosis of a direct carotid cavernous fistula (CCF) was confirmed by CT angiography. CT Angiography showed direct fistulous communication of cavernous segment of right internal carotid artery with right cavernous sinus (Type A right CCF) and linear minimally displaced fracture of right zygomatic arch and linear undisplaced fracture of wall of right orbit. The patient underwent right pterional craniotomy and transcavernous occlusion of fistula of right internal carotid artery (ICA). Surgery was performed with gradual resolution of the symptoms and normalization of the intraocular pressure.

The pathogenesis, symptomatology, treatment of carotid cavernous fistulas are discussed.

Key Words: carotid cavernous fistula, endovascular occlusion, internal carotid artery, open Surgery

(dural), because fistulas to the cavernous sinus arise from dural arteries and not directly from the internal carotid artery. Direct CCF (Type A) usually occurs in young men secondary to trauma. Indirect CCFs (dural CCF) usually occur in postmenopausal, hypertensive

women arising from dural branches of either internal carotid artery (ICA) (Type B) or external carotid artery (ECA) (Type C) or both (mixed or Type D).

Patients often complain of a swollen redeye, orbital pain, diplopia, headache and progressive vision loss. Common clinical signs include proptosis, orbital bruit, chemosis, extraocular muscle palsy, pulsating exophthalmos, ptosis, elevated intraocular pressure, anterior segment ischaemia and retinopathy. ${ }^{6}$ 


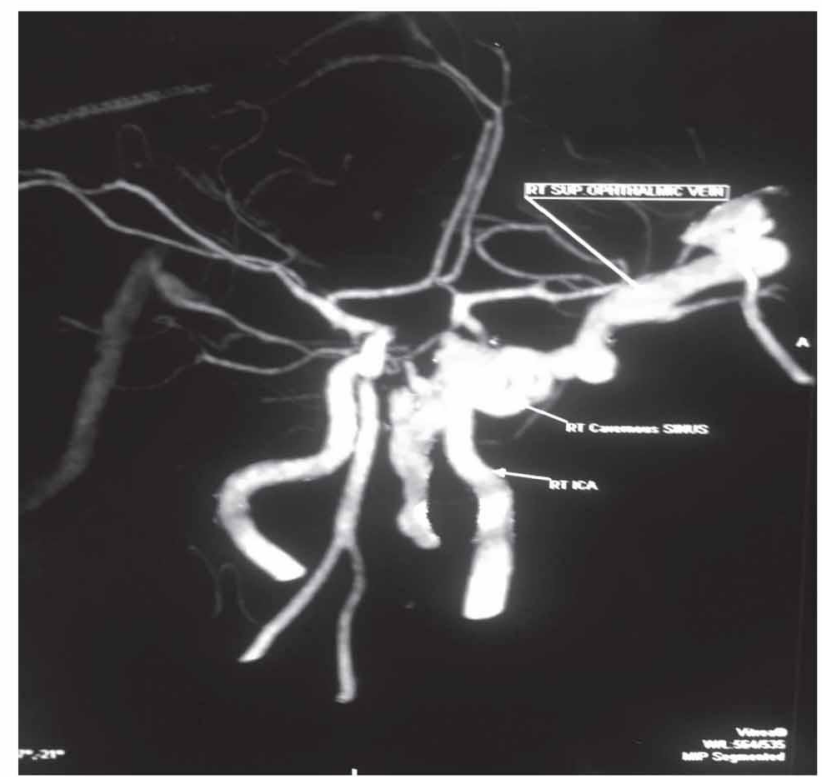

Figure 1: CT angiography demonstrating fistulas tract of carotid cavernous fistula with dilated right superior ophthalmic vein.

In fact, direct CCF are usually caused by severe head injuries or rupture of cavernous aneurysms. In addition, they have also been linked with other surgical trauma such as rhinoplasty, orbital floor fractures reductions, partial maxillectomy, nasopharyngeal biopsy and Le Fort 1 osteotomy. ${ }^{8}$

\section{Case Report}

A previously healthy 18-year-right handed girl had a history of fall from tree around 15 feet height and sustained injury over the face.

She had fracture of right mandible and underwent open reduction and fixation of the right mandibular fracture. Three days later, she developed a painful red eye at the right side, swelling of the eyelids which was slowly progressive initially and associated with the humming pulsating sound and photophobia. After two days, she experienced diplopia and she was referred to our OPD from ophthalmology clinic. The visual acuity was $6 / 6$; also color vision with the Ishihara pseudo isochromatic plates was normal.

There was a striking right sided periorbital edema and ptosis, a bruit was audible over the right orbit. Ocular motility testing revealed an abduction, elevation and depression deficit of the right eye. Pupillary reflexes were normal. Fundoscopy showed slightly dilated vessels. The diagnosis of a traumatic direct CCF was considered.

CT Angiography showed direct fistulous communication of cavernous segment of right internal carotid artery with right cavernous sinus. Right sided

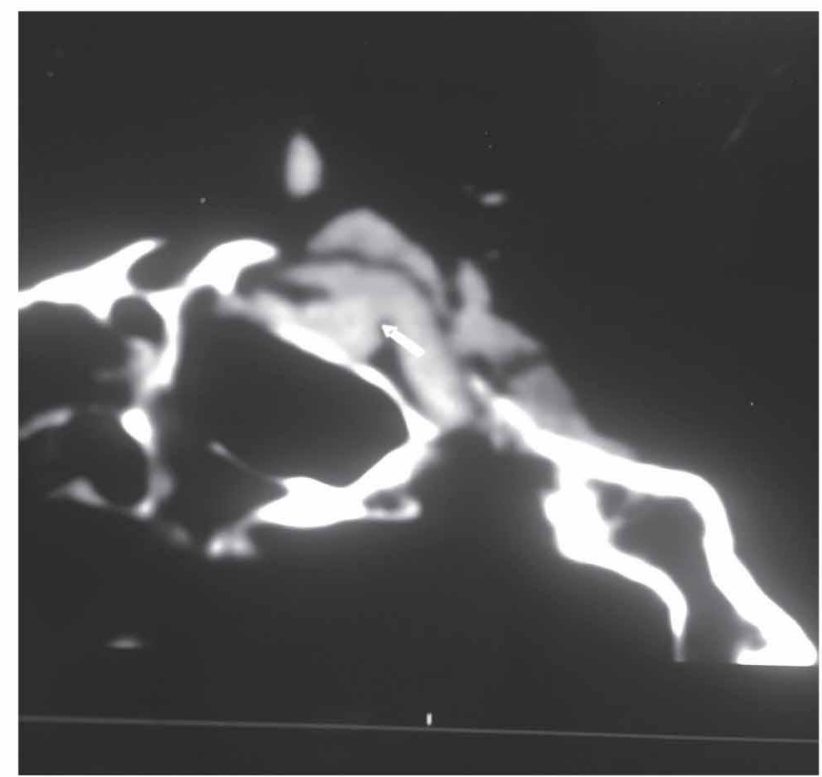

Figure 2: CT angiography demonstrating fistulas tract of carotid cavernous fistula.

Type A CCF was diagnosed (Figure 1, 2). Intracerebral perfusion and collateral circulation showed no anomalies.

The patient underwent right pterional craniotomy, opening of right cavernous sinus at Parkinson's triangle and occlusion of fistula using muscle, muslin gauze and fibrin glue under operating microscope (Figure 3). Initially there was no change on right eye, but after one week symptoms gradually disappeared.

The patient was discharged 10 days after surgery. At the outpatient clinic two months later, all symptoms had disappeared and her right eye came to normal condition.

\section{Discussion}

Barrow's classification of CCF is based on the pattern of arterial supply. ${ }^{1}$ Barrow type A (direct) usually present a single connection between the internal carotid artery and the ipsilateral cavernous sinus. This type of fistula usually show high flow and it is frequently caused by facial trauma. In contrast, indirect CCF are divided into 3 types: B, C and D. In this context, Barrow type B usually shows a connection between meningial branches of the internal carotid and the sinus. Barrow type $\mathrm{C}$ is characterized by connections between meningial branches of the external carotid and the sinus. Finally, Barrow type $\mathrm{D}$ is characterized by connections between meningial branches of the internal and external carotid and the sinus. Contrary to what has been stressed before about direct fistulas, indirect fistulas are low flow dural fistulas. This is an important assertion. In fact, in several cases, indirect fistulas may disappear spontaneously. ${ }^{1}$ Specifically, this 


\section{Sharma et al}

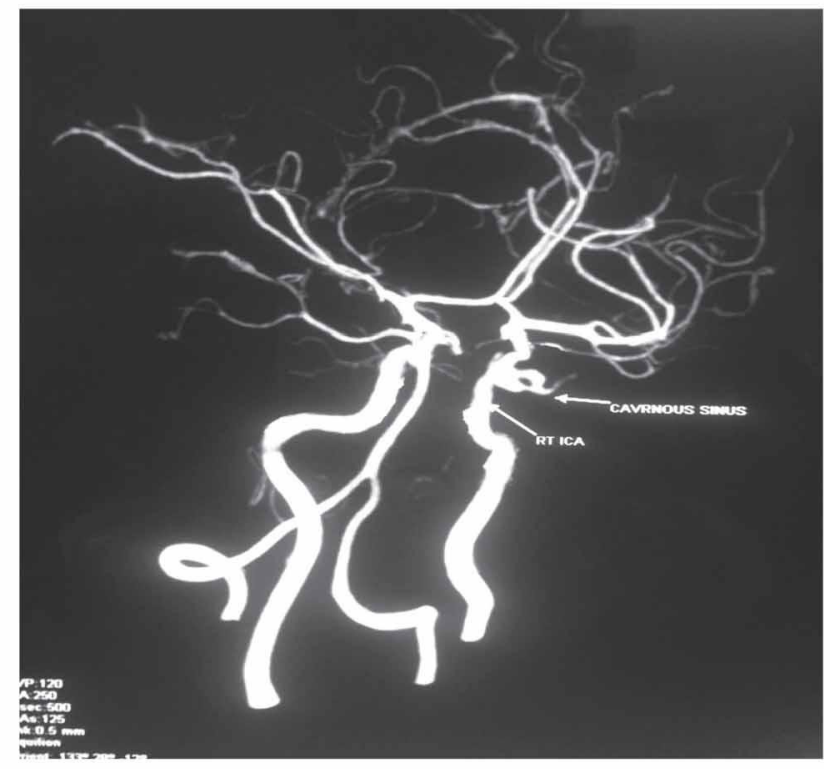

Figure 3: Postoperative CTangiographydemonstrating occlusion of carotid cavernous fistula

type of fistulas could not present clinical manifestations; whereas, direct fistulas usually provoke multiple ocular complications .In this line, we want to emphasize that the ocular complications of carotid-cavernous fistulas are extremely varied. In fact, the list of possible ocular complications includes: pulsating exophthalmos, conjunctival chemosis, orbital blow, diplopia and decrease of vision. ${ }^{2}$ Specifically, the severity of these symptoms depends on the venous drainage capacity and on the intensity of flow.

Traumatic CCFs are usually high flow and direct type fistulas with sudden onset of symptoms. They predominantly occur in young men because of higher incidence of trauma in this population. Underlying mechanisms are direct injury from the skull base fracture or injury from torsion or stretching of the carotid siphon upon impact and impingement of the vessel on bony prominences. Direct CCFs following surgical procedures such as endoscopic nasal surgery and vascularneurosurgery or spontaneously from aneurysm rupture have also been reported. Spontaneous CCF occurs secondary to hypertension, atherosclerosis, neurofibromatosis, and collagen vascular disorders. Bilateral CCF cases comprise $12-15 \%$ of all and are usually indirect in variety. ${ }^{3}$

CCFs drain toward anterior via ophthalmic veins, inferior via pterygoid plexus and inferior petrosal sinus (IPS), contralateral via intercavernous connections, posterior via deep venous system, superior petrosal sinus (SPS), and cerebellar veins, and superior via superficial middle cerebral vein (SMCV). Mostly patients present with orbital symptoms secondary to anterior drainage but clinical presentation may change according to venous drainage pattern. Less commonly, headache, altered mental status, and other neurological deficits such as ischemia or infarction secondary to venous hypertension or steal phenomena may occur. ${ }^{3}$

Patients with a direct Type A fistula generally present with varied complaints, including unilateral visual loss, proptosis, lid swelling, pulsatile tinnitus and/or diplopia. A triad of clinical findings has been described as exophthalmos, orbital bruit, and dilated conjunctival vessels. Clinical findings include venous congestion of the eyelids, conjunctiva and episcleral vessels, cranial nerve palsies, visual loss, proptosis, elevated intraocular pressure, optic disc edema, and dilated and tortuous retinal vessels. ${ }^{10}$

Complications include vision loss and, in rare cases, ischemic ocular necrosis. Non ocular symptoms are though fatal are less common and include epistaxis, subarachnoid hemmorhage and intracerebral hemmorhage due to rupture of fistula

Additionally, with respect to the diagnosis, we want to stress that angiographic study is essential for a proper management of these cases. In fact this test allows a safe diagnosis of carotid-cavernous fistulas. In view of that, cerebral angiography is considered the gold standard for diagnosing these pathologies. Notably, the treatment of carotid-cavernous fistulas has undergone a major evolution over the last years. In fact, the performance of endovascular procedures has radically changed the natural evolution of this diseases. ${ }^{9}$

There are two treatment option for symptomatic CCF either surgery or endovascular. In the past before the availability of endovascular procedure, open surgery was the only one treatment. The fistula is occluded either via superior ophthalmic vein or cavernous sinus. The total occlusion rate of open surgical procedure was more than $80 \%$. But for the last one decade the open surgery for the $\mathrm{CCF}$ is gradually replaced by endovascular procedure because the surgical procedure is technically demanding and associated with high morbidity. Technical difficulties include differentiating ICA from arterialized veins and identifying fistula. ${ }^{4,5}$

\section{Conclusion}

Although endovascular procedure is the best treatment option for symptomatic CCF but open surgery is still an alternative treatment if endovascular procedure is not available, if there is financial constraints or if procedure is repeatedly failed. 


\section{Carotid Cavernous Fistula}

\section{References}

1. Barrow DL, Spector RH, Braun IF, et al. Classification and treatment of spontaneous carotid cavernous fistula. J Neurosurg 62: 248-256, 1985

2. Bojic L, Ivanisevic M, Rogosic V, et al. Traumatic carotid-cavernous fistula. Acta Med Croatica 61: 207-209, 2007

3. Fattahi T, Brandt M, Jenkins W, et al. Traumatic carotid-cavernous fistula: pathophysiology and treatment. The Journal of Craniofacial Surgery 14(2): 240-246, 2003

4. Gemmete J, Ansari S, Gandhi D. Endovascular techniques for treatment of carotid cavernous fistula. Journal of NeuroOphthalmology 29(1): 62-71, 2009

5. Goto K, Hieshima GB, Higashida RT et al. Treatment of direct carotid cavernous sinus fistulae. Various therapeutic approaches and results in 148 cases. Acta radiol Suppl 369: 576-579, 1986

6. Low HL, Sawlani V, Redfern RM. Carotid-cavernous fistula after blunt traumatic to the neck. $\mathbf{B r} \mathbf{J}$ Neurosurg 20: 254-259, 2006

7. Niamtu J ,Campbell RL. Carotid cavernous fistula. J Oral Maxillo fac Surg 40 : 52-56,1982

8. Precious DS. Management of complications of maxillary osteotomies. J Oral Maxillofac Surg Online September, 2004

9. Teng MM, Chang CY, Chiang JH. Double-balloon technique for embolization of carotid cavernous fistulas. Am J Neuroradiol 21:1753-1756, 2000

10. Uysal E, Ulusay M, Basak M, et al. Endovascular trapping of direct carotid cavernous fistula. J Clin Neurosci 17(3) : 392-394, 2010 